\title{
Electronic properties of a carbon nanotube in a field-effect transistor structure: A first-principles study
}

\author{
Kazuyuki Uchida ${ }^{1,2}$ and Susumu Okada ${ }^{2,3}$ \\ ${ }^{1}$ Department of Applied Physics, University of Tokyo, 7-3-1 Hongo, Bunkyo-ku, Tokyo 113, Japan \\ ${ }^{2}$ CREST, Japan Science and Technology Agency, 4-1-8 Honcho, Kawaguchi, Saitama 332-0012, Japan \\ ${ }^{3}$ Center for Computational Sciences, University of Tsukuba, 1-1-1 Tennodai, Tsukuba 305-8577, Japan \\ (Received 14 March 2008; revised manuscript received 8 September 2008; published 3 February 2009)
}

\begin{abstract}
A first-principles electron-state calculation is performed to explore the electronic properties of a semiconductor carbon nanotube (CNT) in a field-effect transistor structure. Field-effect electron/hole doping results in a carrier distribution spread over the whole $\mathrm{C}-\mathrm{C}$ network of the CNT, while accumulated charges, that explain the electrostatic capacitance $C_{0}$ between the $\mathrm{CNT}$ and gate electrode, are concentrated in a small part of the CNT facing the gate electrode. We also demonstrate that the density of states structure of the CNT gives substantial bias dependences to the total capacitance $C$ of the system.
\end{abstract}

DOI: $10.1103 /$ PhysRevB.79.085402

PACS number(s): 73.22.-f, 71.15.-m, 73.63.Fg, 77.84.- $\mathrm{s}$

\section{INTRODUCTION}

In the past decade, carbon nanotubes (CNTs) (Ref. 1) have attracted a great deal of attention in both the pure and applied sciences due to their unique structural and electronic properties. $^{2-4}$ Their fascinating features open the possibility of fabricating superior nanometer-scale electronic devices, in which the CNTs are key constituent units with conventional materials. Indeed, it has been demonstrated that individual semiconductor CNTs act as field-effect transistors (FETs), ${ }^{5-8}$ where the CNT composes a hybrid structure with a gate electrode and an intermediate insulator, bridging between the source and drain electrodes as shown in Fig. 1(a). A current flow along the CNT axis is controlled by applying a gate-bias voltage $\mu$ to the capacitor between the CNT and the gate electrode.

Knowledge about the characteristics of the CNT-FETs is accumulating steadily from experiments. However, little is known of the fundamentals of the electronic properties of the CNTs in the FET structures. Though several model calculations reported the qualitative properties of CNT-FETs (Refs. 9 and 10) and density-functional calculations are performed for coaxial-cylindrical geometries, ${ }^{11-13}$ it is still unclear how the electronic properties of the CNTs are modulated under an electric field in a capacitor structure consisting of the CNT placed over the gate electrode. In particular, quantitative discussions on the distributions of carriers injected by a gatebias application and the capacitance between the CNT and the planer gate electrode have not yet been addressed from first principles. The purpose of this work is to unravel these two issues closely concerned with the fundamental function of the CNT-FETs and to provide theoretical knowledge on the electronic properties of the CNT in the CNT-FET structures by using a first-principles electron-state calculation.

In the present work, we perform a first-principles electronic-structure calculation of a semiconducting $(7,0)$ CNT placed above a gate electrode. Our calculations reveal that the field-effect doping results in a carrier distribution, i.e., highest-occupied (HO) or lowest-unoccupied (LU) state wave functions just on the Fermi level, spread over the whole C-C network of the CNT. This indicates that the cur- rent between the source and drain electrodes flows through the whole cross section of a CNT. In sharp contrast, the accumulated charges in the CNT, which determines the electrostatic capacitance of the system, are found to be concentrated in a small part of the CNT facing the gate electrode, according to the dielectric polarization of all of the electron states below the Fermi level. Further analyses clarify that the total capacitance of the CNT-FET system strongly depends on the density of states (DOS) structure of the CNT.

\section{MODEL AND METHOD}

We simulate a $(7,0)$ CNT capacitor with a planer gate electrode. Figure 1(b) shows our structural model. The gate electrode is modeled by a uniformly charged sheet with $0.2 \AA$ thickness. The gate electrode is separated from the

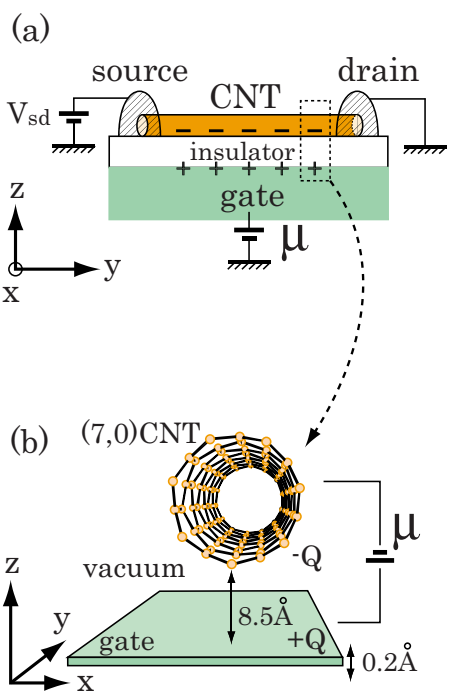

(c)

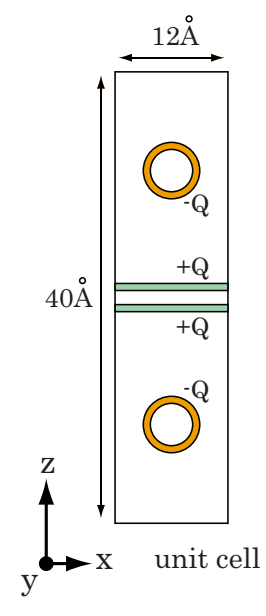

FIG. 1. (Color online) (a) A schematic of a CNT-FET. (b) Our model of the capacitor between a $(7,0) \mathrm{CNT}$ and the gate electrode. In our model, the intermediate insulator between the CNT and the gate electrode is replaced with a vacuum. (c) The unit cell for our actual calculations under the three-dimensional periodic boundary condition. 
CNT by an $8.5 \AA$ vacuum region in our model. The $(7,0)$ CNT is semiconducting before the carrier injections and its band-gap width calculated is $\epsilon_{G}=0.23 \mathrm{eV}$. To simulate the field-effect carrier doping, we inject extra electrons/holes $(\mp Q)$ into the CNT and distribute counter charges $( \pm Q)$ on the gate electrode. A gate electrode with a constant potential and an infinite DOS value is approximately described by this model. The extra electrons/holes shift the electrostatic potential level of the CNT and that of the gate electrode in opposite directions, so that a gradient of the potential, corresponding to the externally applied electric field, exists in the vacuum region between the $\mathrm{CNT}$ and the gate electrode. We calculate the distribution of the carriers $\rho_{\epsilon_{F}}(r)$ and that of the accumulated charges $\Delta \rho_{-Q}(r)$ under this electric field. The electric field is perfectly screened by the CNT in our model, as we put exactly the opposite charge on the $\mathrm{CNT}(\mp Q)$ and on the gate electrode $( \pm Q)$.

We use the three-dimensional periodic boundary condition in the present work. We place a pair of CNT-electrode systems back to back in the unit cell [Fig. 1(c)] to eliminate dipole-dipole interactions among the cells induced by the stacking structure of the charged CNT $(-Q)$ intercalated by the charged electrodes $(+Q)$. A CNT and its mirrors are separated by at least $7 \AA$ in the $x$ direction and $12 \AA$ in the $z$ direction, which ensures negligible wave-function overlapping between the neighboring CNTs. We perform a firstprinciples electron-state calculation with the local-density approximation (LDA) (Refs. 14 and 15) in the framework of the density-functional theory (DFT). ${ }^{16}$ We use ultrasoft pseudopotentials ${ }^{17}$ and a plane-wave basis set with the cutoff energy chosen as 36 Ry. The Kohn-Sham equation ${ }^{18}$ is solved by using the conjugate-gradient (CG) minimization technique. Ionic configurations are also optimized by performing the CG minimization at $Q=0$ (zero-bias condition). For the charged states, we fix the atomic structure of the CNT as that obtained under the zero-bias voltage. All the computations have been done using the TAPP program package.${ }^{19}$ We use the notation of $e=1$ in the following descriptions.

\section{RESULTS AND DISCUSSIONS}

Figures 2(a) and 2(b) show the carrier distributions $\rho_{\epsilon_{F}}(r)$ on the CNT, projected onto the cross section perpendicular to the tube axis. The CNT is injected with $-Q=-1.8$ $\times 10^{-3} \mathrm{e} /$ atom extra electrons in Fig. 2(a) and 1.8 $\times 10^{-3} \mathrm{e} /$ atom holes in Fig. 2(b), respectively. The corresponding bias voltage $\mu$ between the CNT and the gate electrode is (a) $1.59 \mathrm{~V}$ and (b) $-1.56 \mathrm{~V}$ for the electron and hole dopings, respectively. The carrier distribution corresponds to the charge density near the CNT's Fermi level $\epsilon_{F}$ and is calculated by

$$
\rho_{\epsilon_{F}}(r)=\sum_{n, \mathbf{k}}\left|\psi_{n, \mathbf{k}}(r)\right|^{2} \delta\left(\epsilon_{n, \mathbf{k}}-\epsilon_{F}\right) .
$$

The results indicate that the conducting channels along the tube axis, which is opened under the field-effect carrier doping, are spread over the whole C-C network of the CNT almost isotropically, although the electric field exists be-

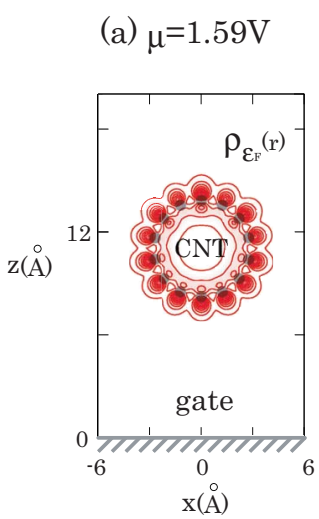

(b) $\mu=-1.56 \mathrm{~V}$

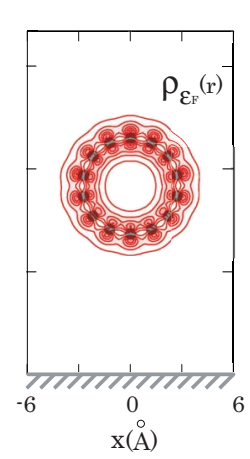

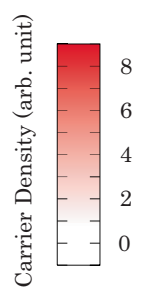

FIG. 2. (Color online) Distribution of the carriers $\rho_{\epsilon_{F}}(r)$ under a finite gate-bias voltage $\mu$, projected onto the cross section perpendicular to the CNT axis. Black circles denote carbon atoms. The results indicate that the current between the source and drain electrodes flows through the whole cross section of a CNT. We calculate $\rho_{\epsilon_{F}}(r)$ as the charge density near the Fermi level. The gate-bias voltage between the CNT and the electrode is (a) $\mu=1.59 \mathrm{~V}$ and (b) $\mu=-1.56 \mathrm{~V}$, respectively.

tween the CNT and the gate electrode when $Q \neq 0$.

Left panels in Figs. 3(a)-3(c) show the DOS spectrum of the CNT together with the Fermi level of the CNT $\epsilon_{F}$ and that of the gate electrode $\epsilon_{F}^{\text {gate }}$, under the gate-bias voltage of (a) $\mu=1.59 \mathrm{~V}$, (b) $\mu=-1.56 \mathrm{~V}$, and (c) $\mu=0$, respectively. Here, $\mu$ is defined by $\mu=\epsilon_{F}-\epsilon_{F}^{\text {gate }}$. We assume that the Fermi levels of the CNT $\left(\epsilon_{F}\right)$ and that of the gate electrode $\left(\epsilon_{F}^{\text {gate }}\right)$ are located at the middle of the band gap of the CNT when $\mu=0$ [Fig. 3(c)]. For all the charged states [Figs. 3(a)-3(c)], energies are measured from the Fermi level $\epsilon_{F}^{\text {gate }}$ of the gate electrode placed at $z=0$. Since the electron/hole injection into the CNT shifts the Fermi level $\epsilon_{F}$ of the CNT upwards/ downwards into the lowest/highest branch of the conduction/ valence band [Figs. 3(a) and 3(b)], the distributions of the carriers $\rho_{\epsilon_{F}}(r)$ reflect the wave functions of the LU state in Fig. 2(a) and the HO state in Fig. 2(b), respectively. Even under the bias voltage, the electronic structure of the CNT rather preserves their characteristics at the zero-bias voltage, which results in the carrier distribution spread over the whole C-C network of the CNT almost isotropically. Indeed, the calculated DOS under the gate-bias voltage $\mu$ of about $\pm 1.6 \mathrm{~V}$ exhibits a rigid-band nature ${ }^{20}$ except for their energy shift with respect to the Fermi level of the gate electrode $\epsilon_{F}^{\text {gate }}$, possessing almost the same characteristics as that obtained at the zero-bias voltage [Figs. 3(a)-3(c)].

Figure 4 shows the capacitance $C(\equiv d Q / d \mu)$ between the CNT and the gate electrode, calculated as a function of the bias voltage $\mu$. We observe that the capacitance $C(\mu)$ shows substantial bias dependences. The capacitance $C$ is zero when the bias $\mu$ is in the range of $-\epsilon_{G} / 2<\mu<\epsilon_{G} / 2$. The capacitance increases steeply when increasing or decreasing the bias voltage, possessing maxima just above $\left(\mu=\epsilon_{G} / 2\right)$ and below $\left(\mu=-\epsilon_{G} / 2\right)$ the zero region. Further increasing ( $\left.\mu>\epsilon_{G} / 2\right)$ or decreasing $\left(\mu<-\epsilon_{G} / 2\right)$ the bias voltage, the capacitance decreases gradually. The maxima of the capacitance $C$ corresponds to the Fermi level $\epsilon_{F}$ just on the van Hove singularities in the DOS spectrum, and the band-gap 


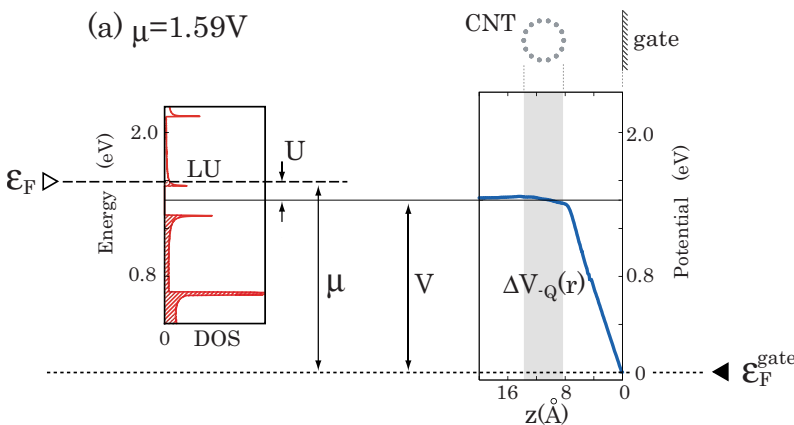

(b) $\mu=-1.56 \mathrm{~V}$

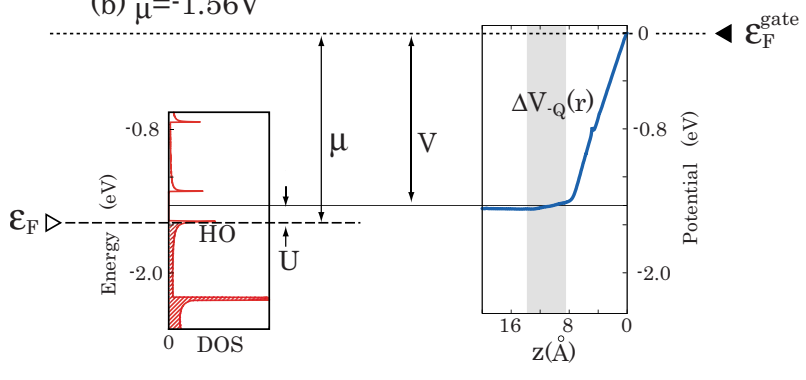

(c) $\mu=0$

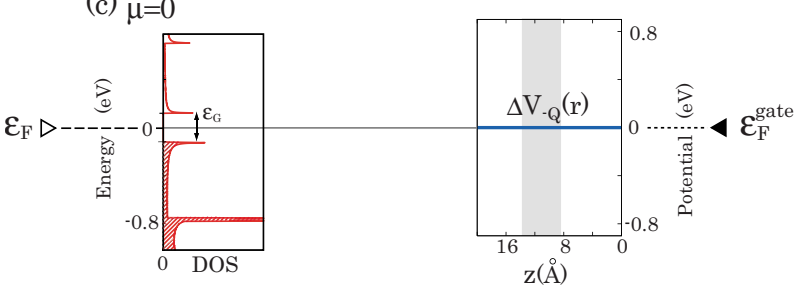

FIG. 3. (Color online) Density of states structure of the CNT (left panels) together with the bias-induced potential (right panels) $\Delta V_{-Q}(r)$ along the $z$ direction, under the gate-bias voltage of (a) $\mu=1.59 \mathrm{~V}$, (b) $\mu=-1.56 \mathrm{~V}$, and (c) $\mu=0$. Open and filled triangles denote the Fermi-level positions of the CNT $\epsilon_{F}$ and the gate electrode $\epsilon_{F}^{\text {gate }}$, respectively. Energies are measured from the Fermi level of the gate electrode $\epsilon_{F}^{\text {gate }}$ in these figures. The shaded range in each right panel corresponds to the position of the CNT.

width $\epsilon_{G}(=0.23 \mathrm{eV})$ of the $(7,0)$ CNT coincides with the width of the zero-capacitance region. These results infer that the capacitance $C(\mu)$ reflects the electronic structures of the CNT besides the usual electrostatic capacitance $C_{0}$.

Actually, as shown in the left and right panels in Figs. 3(a) and 3(c) [Figs. 3(b) and 3(c)], the bias voltage $\mu$ consists of an energy shift $(U)$ of the CNT's Fermi level $\epsilon_{F}$ within the DOS spectrum of the CNT after the electron/hole $(-Q)$ accommodation and an electrostatic energy shift $(V)$ of the CNT's whole DOS spectrum with respect to the Fermi level $\epsilon_{F}^{\text {gate }}$ of the gate electrode

$$
\mu=U+V
$$

This electrostatic shift $V$ of the whole DOS spectrum is consistent with the potential shift $\Delta V_{-Q}(r)$ induced between the CNT and the gate electrode in the sense that

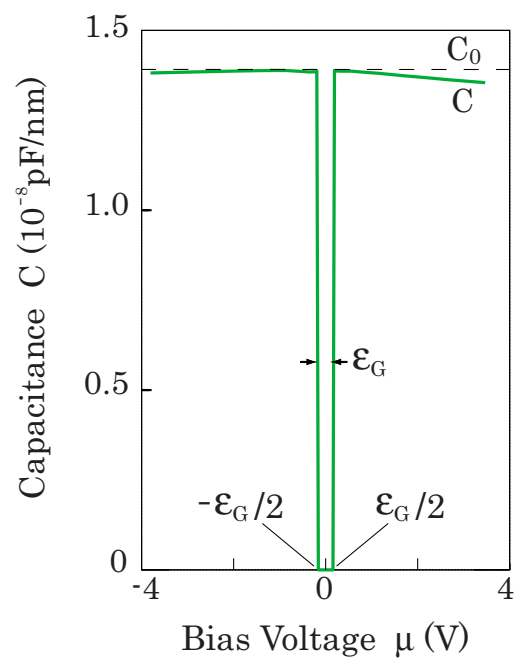

FIG. 4. (Color online) Capacitance $C(=d Q / d \mu)$ between the $(7,0) \mathrm{CNT}$ and the gate electrode, calculated as a function of the bias voltage $\mu\left(=\epsilon_{F}-\epsilon_{F}^{\text {gate }}\right)$. We show the capacitance per unit length along the CNT axis. The capacitance $C$ shows substantial bias dependences, reflecting the DOS structure $D$ of the CNT as $1 / C$ $=1 / C_{0}+1 / D$. The dashed line indicates the electrostatic capacitance $C_{0}$. The total capacitance $C$ is smaller than the $C_{0}$ value due to the contributions from $D$. We note that $\epsilon_{G}(=0.23 \mathrm{eV})$ corresponds to the band-gap width of the CNT.

$$
V \sim \frac{\int d r\left[\Delta V_{-Q}(r) \rho_{-Q}(r)\right]}{\int d r\left[\rho_{-Q}(r)\right]} .
$$

Here, $\rho_{-Q}(r)$ is the total electron density in the CNT and $\Delta V_{-Q}(r)$ is the induced potential calculated by $\Delta V_{-Q}(r)$ $=V_{-Q}(r)-V_{0}(r)$, where $V_{-Q}(r)$ and $V_{0}(r)$ are the total potentials with and without injecting the extra electrons/hole into the CNT, respectively. The right panels of Figs. 3(a)-3(c) show the induced potential $\Delta V_{-Q}(r)$, calculated under the gate-bias voltage of (a) $\mu=1.59 \mathrm{~V}$, (b) $\mu=-1.56 \mathrm{~V}$, and (c) $\mu=0$, respectively. As the electrostatic component is dominant in $\Delta V_{-Q}(r)$, we can regard $V$ as the electrostatic shift. A slight influence of the exchange-correlation potential can be observed as a small singular behavior of $\Delta V_{-Q}(r)$ at $z \sim 4 \AA$ in the right panels of Fig. 3(a) and 3(b).

From Eq. (2), the capacitance $C$ is divided $a^{23}$

$$
\frac{1}{C}=\frac{d \mu}{d Q}=\frac{d(U+V)}{d Q}=\frac{1}{D}+\frac{1}{C_{0}} .
$$

We thus understand that the DOS of the CNT at the Fermi level, $D(=d Q / d U)$, indeed contributes to the total capacitance $C$. This explains the substantial bias dependences of the capacitance observed in Fig. 4. The electrostatic capacitance $C_{0}\left(=d Q / d V \sim 1.4 \times 10^{-8} \mathrm{pF} / \mathrm{nm}\right)$, which shows almost no bias dependences, is also drawn by a dashed line in Fig. 4. From Eq. (4), we summarize the asymptotic behavior of the capacitance. In a case of a large DOS limit, corresponding to the Fermi level $\epsilon_{F}$ of the CNT at the van Hove singularities, the capacitance $C$ is almost composed of the 
(a) $\mu=1.59 \mathrm{~V}$

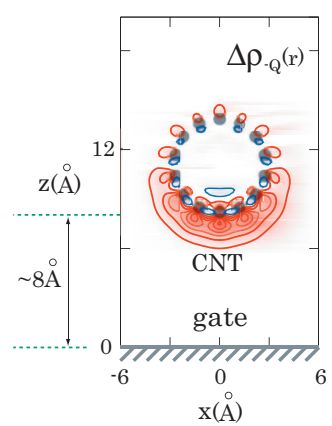

(b) $\mu=-1.56 \mathrm{~V}$

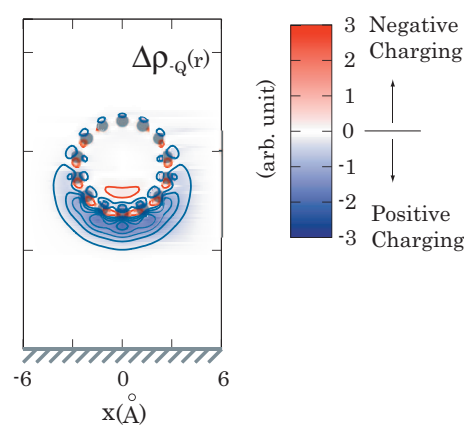

FIG. 5. (Color) Distribution of the accumulated charge $\Delta \rho_{-Q}(r)$, projected onto the cross section perpendicular to the CNT axis. Black circles denote carbon atoms. This distribution of $\Delta \rho_{-Q}(r)$ determines the electrostatic capacitance $C_{0}$ between the CNT and the gate electrode. We calculate $\Delta \rho_{-Q}(r)$ by $\Delta \rho_{-Q}(r)=\rho_{-Q}(r)$ $-\rho_{0}(r)$, where $\rho_{-Q}(r)$ and $\rho_{0}(r)$ are the total electron densities below the Fermi level, calculated with and without injecting extra electrons/holes into the CNT. The corresponding gate-bias voltage is $\mu=1.59 \mathrm{~V}$ in (a) and $\mu=-1.56 \mathrm{~V}$ in (b), respectively. The difference between the distribution of the accumulated charge $\Delta \rho_{-Q}(r)$ (Fig. 5) and that of the carrier $\rho_{\epsilon_{F}}(r)$ (Fig. 2) is ascribed to the dielectric polarization of all of the electron states below the Fermi level, contributing only to $\Delta \rho_{-Q}(r)$.

electrostatic capacitance $C_{0}$. On the other hand, for a small DOS region, the DOS value $D$ is dominant in determining the capacitance $C$, so that the capacitance $C$ is remarkably sensitive to the DOS spectrum.

The electrostatic capacitance $C_{0}(=d Q / d V)$ also gives a qualitative discussion on the distribution of the accumulated charge in the CNT through a comparison to a parallel-plate capacitor in the classical electromagnetism. In a parallelplate capacitor, an interplate distance of $d \sim 8 \AA$ results in the electrostatic capacitance of $1.4 \times 10^{-8} \mathrm{pF} / \mathrm{nm}$, which is an identical value with that of the present CNT-FET $\left(\equiv C_{0}\right)$. As the axis of the CNT is located at $\sim 11 \AA(>d \sim 8 \AA)$ from the gate electrode in our model (see Fig. 5), it is suggested that the accumulated charge is substantially concentrated in a small part of the CNT facing the gate electrode. To corroborate this point, in Fig. 5, we show the distribution of the accumulated charge $\Delta \rho_{-Q}(r)$ calculated by

$$
\Delta \rho_{-Q}(r)=\rho_{-Q}(r)-\rho_{0}(r),
$$

where $\rho_{-Q}(r)$ and $\rho_{0}(r)$ are the total electron densities with and without extra electron/hole injections into the CNT, respectively. The bias voltage $\mu$ between the CNT and the gate electrode is $1.59 \mathrm{~V}$ in Fig. 5(a) and $-1.56 \mathrm{~V}$ in Fig. 5(b), respectively. We actually find that $\Delta \rho_{-Q}(r)$ is concentrated in a small part of the CNT facing the gate electrode both when the CNT is doped with electrons [Fig. 5(a)] and holes [Fig. $5(\mathrm{~b})$, respectively. We thus find that these distributions of the accumulated charges indeed explain the calculated value of the electrostatic capacitance $C_{0}$ between the CNT and the gate electrode. The distributions of the accumulated charge $\left[\Delta \rho_{-Q}(r)\right]$ are completely different from the carrier distributions $\left[\rho_{\epsilon_{F}}(r)\right]$ in Figs. 2(a) and 2(b). These distributions of the accumulated charge $\Delta \rho_{-Q}(r)$ also result in a rapid screening in the induced potential $\Delta V_{-Q}(r)$ near the small part of the CNT facing the gate electrode, as is shown in the right panels of Figs. 3(a) and 3(b).

We finally explain why the distribution of the accumulated charge $\Delta \rho_{-Q}(r)$ [Figs. 5(a) and 5(b)] is substantially different from that of the carrier $\rho_{\epsilon_{F}}(r)$ [Figs. 2(a) and 2(b)], though they are obtained simultaneously at the same bias voltage $\mu$. As we have already mentioned, the carrier distribution $\rho_{\epsilon_{F}}(r)$ reflects the wave functions of only the LU and HO states of the CNT around the Fermi level. Conversely, the distribution of the accumulated charge $\Delta \rho_{-Q}(r)$ includes the deformations of the wave functions of all the occupied electron states below the Fermi level that are not directly involved in the extra electron/hole accommodations. Though such a deformation of each one-electron wave function is much smaller than the wave function itself and thus the rigid-band picture holds [Figs. 3(a)-3(c)], the total sum of the deformations, i.e., dielectric polarization, ${ }^{11-13,24}$ is sufficiently large to give the substantial differences between the distribution of the accumulated charge $\Delta \rho_{-Q}(r)$ and that of the carrier $\rho_{\epsilon_{F}}(r)$ in the CNT-FET structures. We have analyzed the contributions to $\Delta \rho_{-Q}(r)$ from each band in detail, finding that the contributions from the $\pi$ bands are more effective than those from the $\sigma$ bands. ${ }^{25}$

\section{CONCLUSION}

In summary, we have investigated the field-effect carrier doping into a $(7,0)$ CNT placed above a gate electrode. Under a finite gate-bias voltage, the carrier distribution $\rho_{\epsilon_{F}}(r)$ remains spread over the whole $\mathrm{C}-\mathrm{C}$ network of the CNT almost isotropically, while the accumulated charge $\Delta \rho_{-Q}(r)$, which explains the electrostatic capacitance $C_{0}$ between the CNT and the gate electrode, is substantially concentrated in a small part of the CNT facing the gate electrode. This difference is ascribed to the dielectric polarization of all of the electron states below the Fermi level, which is contributing only to the latter. We have also demonstrated that the total capacitance $C$ shows substantial bias dependences, reflecting the DOS structures $D$ of the CNT as $1 / C=1 / C_{0}+1 / D$.

\section{ACKNOWLEDGMENTS}

This work was partly supported by CREST-Japan Science and Technology Agency, and a grant-in-aid for scientific research from the Ministry of Education, Culture, Sports, Science, and Technology of Japan. Computations were performed at Yukawa Institute of Theoretical Physics, Kyoto University, Cyber Media Center, Osaka University, Information Synergy Center, Tohoku University, Institute of Solid State Physics, Tokyo University, and Research Center of Computational Science, National Institute of Natural Sciences. 
${ }^{1}$ S. Iijima, Nature (London) 354, 56 (1991).

${ }^{2}$ N. Hamada, S. I. Sawada, and A. Oshiyama, Phys. Rev. Lett. 68 , 1579 (1992).

${ }^{3}$ R. Saito, M. Fujita, G. Dresselhaus, and M. D. Dresselhaus, Appl. Phys. Lett. 60, 2204 (1992).

${ }^{4}$ S. Okada and A. Oshiyama, J. Phys. Soc. Jpn. 72, 1510 (2003).

${ }^{5}$ S. J. Tans, A. R. M. Verschueren, and C. Dekker, Nature (London) 393, 49 (1998).

${ }^{6}$ Y. Ohno, S. Iwatsuki, T. Hiraka, T. Okazaki, S. Kishimoto, K. Maezawa, H. Shinohara, and T. Mizutani, Jpn. J. Appl. Phys., Part 1 42, 4116 (2003).

${ }^{7}$ H. Shimotani, T. Kanbara, Y. Iwasa, K. Tsukagoshi, Y. Aoyagi, and H. Kataura, Appl. Phys. Lett. 88, 073104 (2006).

${ }^{8}$ S. Ilani, L. A. K. Donev, M. Kindermann, and P. L. McEuen, Nat. Phys. 2, 687 (2006).

${ }^{9}$ D. L. John, L. C. Castro, and D. L. Pulfrey, J. Appl. Phys. 96, 5180 (2004).

${ }^{10}$ J. Guo, S. Goasguen, M. Lundstrom, and S. Datta, Appl. Phys. Lett. 81, 1486 (2002).

${ }^{11}$ P. Pomorski, L. Pastewka, C. Roland, H. Guo, and J. Wang, Phys. Rev. B 69, 115418 (2004).

${ }^{12}$ L. Latessa, A. Pecchia, A. Di Carlo, and P. Lugli, Phys. Rev. B 72, 035455 (2005).

${ }^{13}$ K. Uchida, S. Okada, K. Shiraishi, and A. Oshiyama, Phys. Rev. B 76, 155436 (2007).

${ }^{14}$ D. M. Ceperley and B. J. Alder, Phys. Rev. Lett. 45, 566 (1980).

${ }^{15}$ J. P. Perdew, K. Burke, and M. Ernzerhof, Phys. Rev. Lett. 77,
3865 (1996).

${ }^{16}$ P. Hohenberg and W. Kohn, Phys. Rev. 136, B864 (1964).

${ }^{17}$ D. Vanderbilt, Phys. Rev. B 41, 7892 (1990).

${ }^{18}$ W. Kohn and L. J. Sham, Phys. Rev. 140, A1133 (1965).

${ }^{19}$ Tokyo $A b$ initio Program Package (TAPP) is developed by a consortium initiated at University of Tokyo; J. Yamauchi, M. Tsukada, S. Watanabe, and O. Sugino, Phys. Rev. B 54, 5586 (1996); O. Sugino and A. Oshiyama, Phys. Rev. Lett. 68, 1858 (1992).

${ }^{20}$ In general, the shape of the DOS spectrum can be changed by the charge accumulation, accompanied by large structural deformations induced by the charging (Ref. 21), band modifications under extremely large electric fields (Ref. 22), or some exchangecorrelation effect.

${ }^{21}$ K. Uchida, S. Tsuneyuki, and T. Schimizu, Phys. Rev. B 68, 174107 (2003).

${ }^{22}$ J. O'Keeffe, C. Wei, and K. Cho, Appl. Phys. Lett. 80, 676 (2002).

${ }^{23}$ M. Büttiker, J. Phys.: Condens. Matter 5, 9361 (1993). As we assume that the gate electrode has an infinite DOS value in the present work, the DOS of the gate electrode does not contribute to the total capacitance of the system. See also (Ref. 13).

${ }^{24}$ L. X. Benedict, S. G. Louie, and M. L. Cohen, Phys. Rev. B 52, 8541 (1995).

${ }^{25}$ The magnitude of the contribution to $\Delta \rho_{-Q}(r)$ from each band was measured by $\int z \Delta \rho_{-Q}^{n}(r) d r$, where $\Delta \rho_{-Q}^{n}(r)$ is the change in the charge density of the $n$th band induced by the charging. 